\title{
SMALL STOCHASTIC PERTURBATIONS IN A GENERAL FRACTIONAL KINETIC EQUATION *
}

\author{
DAVID MÁrquez-CARreras ${ }^{1}$
}

\begin{abstract}
In this paper we study some properties of the density for the law of the solution to a generalized multidimensional fractional kinetic equation driven by a Gaussian noise, white in time and correlated in space. The diffusion operator is the composition between the Bessel and Riesz potentials with any fractional parameters. We also establish Varadhan's estimates for the solution to the equation obtained by perturbing the noise.
\end{abstract}

Mathematics Subject Classification. 60G60, 60H15, 60H30, 60G10, 60G15, 60H07.

Received September 10, 2013. Revised January 21, 2014.

\section{INTRODUCTION}

We deal with the following kind of stochastic partial differential equations

$$
\partial_{t} u^{\varepsilon}(t, x)+(I-\Delta)^{\frac{\alpha}{2}}(-\Delta)^{\frac{\beta}{2}} u^{\varepsilon}(t, x)=\varepsilon \theta\left(u^{\varepsilon}(t, x)\right) \dot{W}(t, x), \quad \varepsilon>0,
$$

where $t \in \mathbb{R}_{+}, x \in \mathbb{R}^{d}, d \in \mathbb{N}, \alpha \geq 0$ and $\beta>0$. Initially we assume that $\theta: \mathbb{R} \rightarrow \mathbb{R}$ is a Lipschitz continuous function and the process $\dot{W}$ is a Gaussian noise, white in time and correlated in space. We will specify later the required conditions on the function $\theta$ and the process $\dot{W}$. The initial conditions are null for the sake of simplicity. Moreover, $I$ and $\Delta$ are the identity and Laplacian operators on $\mathbb{R}^{d}$, respectively. Then, the integral operators $(-\Delta)^{\beta / 2}$ and $(I-\Delta)^{\alpha / 2}$ have to interpreted as the inverse of the Riesz and Bessel potentials, respectively. These types of operators are widely studied in Samko et al. [35], Stein [37] and Angulo et al. [3].

This kind of generalized fractional kinetic equation was introduced to model some physical phenomena such as diffusion in porous media with fractal geometry, kinematics in viscoelastic media, relaxation processes in complex systems, propagation of seismic waves, anomalous diffusion, turbulence, neurophysiology, ecology, hydrology, image analysis, air pollution, economics and finance, etc. For more information and details on these modelings we refer the reader to Anh and Leonenko [4] and Angulo et al. [2] and the references therein. Moreover, this sort of stochastic partial differential equations is a generalization of a lot of classical and well-known equations as, for exemple, the stochastic heat equation or higher-order parabolic equations.

Keywords and phrases. Stochastic fractional kinetic and heat equation, Bessel and Riesz potentials, small perturbations, density estimates, Malliavin calculus.

* Partially supported by the Grant MTM2013-31192. Universitat de Barcelona

1 Departament de Probabilitat, Lògica i Estadística, Facultat de Matemàtiques, Universitat de Barcelona, Gran Via 585, 08007 Barcelona, Espagne. davidmarquez@ub.edu 
Recently, several authors have studied this kind of stochastic equations and similar others from a mathematical point of view. In Angulo et al. [2] the authors have linked this type of equation to the Eulerian theory of turbulent dispersion. Indeed, the concentration field $u(t, x)$ is commonly assumed to satisfy the advectiondiffussion equation

$$
\partial_{t} u+\nabla \cdot(v u)=\kappa \Delta u, \quad t \in \mathbb{R}_{+}, x \in \mathbb{R}^{d},
$$

where $\nabla$ is the gradient vector, $v(t, x)$ is the velocity vector field and $\kappa$ is the molecular diffusivity. The assumptions of this model are equivalent to supposing that the particle trajectories $x(t)$ satisfy the Itô stochastic differential equation

$$
\mathrm{d} s=u(t, x) \mathrm{d} t+(2 \kappa)^{\frac{1}{2}} d B,
$$

where the components of $d B$ are the increments of independent Brownian motions. The long-range dependence is a basic issue in modelling observed data in a large number of fields given just before. An important example is the fractional Brownian motion with Hurst index $H \in\left(\frac{1}{2}, 1\right)$ (see, for instance, Mandelbrot and Van Ness [23]).

Anh and Leonenko [5] have studied (1.1) with $\theta=0$ and a measurable random field as an initial condition. They have presented a renormalization and homogenization theory for this sort of equation. In [34], an exponential function of the fractional Riesz-Bessel motion was considered as the initial condition. We also mention the papers $[2,4]$ where the authors deal with a more general type of operators:

$$
\frac{\partial^{\eta}}{\partial t^{\eta}}+\mu\left[(I-\Delta)^{\frac{\alpha}{2}}(-\Delta)^{\frac{\beta}{2}}\right], \quad \mu>0, \eta \in(0,1], \alpha \geq 0, \beta>0 .
$$

If the reader is interested in this subject we give a long list of references about it: $[1-5,7-9,13,16,19-22,25,29]$.

Using the ideas giving by Dalang and Frangos ([10,11]), we will understand a solution of $(1.1)$ to be a jointly measurable adapted process satisfying the integral form

$$
u^{\varepsilon}(t, x)=\varepsilon \int_{0}^{t} \int_{\mathbb{R}^{d}} K_{t-s}(x-y) \theta\left(u^{\varepsilon}(s, y)\right) W(\mathrm{~d} s, \mathrm{~d} y),
$$

where $K$ is the fundamental solution of (1.1), this means that it is the solution of

$$
\partial_{t} K_{t}(x)+(I-\Delta)^{\alpha / 2}(-\Delta)^{\beta / 2} K_{t}(x)=0,
$$

and $\left\{W_{t}(A), t \in \mathbb{R}_{+}, A \in \mathcal{B}_{b}\left(\mathbb{R}^{d}\right)\right\}$ is a worthy martingale measure builded from the initial Gaussian process. $\mathcal{B}_{b}\left(\mathbb{R}^{d}\right)$ are the bounded Borel subsets of $\mathbb{R}^{d}$. This solution (1.2) is proved assuming a square-integrability condition on the Fourier transform of the fundamental solution $K$. All these aspects will be given in more detail in the following section. There are many papers related to this formulation, as for example $[10,11,26,30,33]$, etc.

In Márquez-Carreras [25], assuming some more general hypothesis on $\theta$, we have proved that the law of $u^{\varepsilon}(t, x)$ is absolutely continuous with respect to Lebesgue's measure on $\mathbb{R}$ and its density is infinitely differentiable. Denote by $p_{t, x}^{\varepsilon}(t, x)$ the density for the law of the process $u^{\varepsilon}(t, x)$, solution to (1.1). In this paper we will show that this density is Hölder continuous in time and in space. More specifically, following the ideas giving in [27], we will check that the density satisfies:

$$
\begin{aligned}
\left|p^{\varepsilon}(t, x)-p^{\varepsilon}(s, x)\right| & \leq C|t-s|^{\sigma_{1}}, \\
\left|p^{\varepsilon}(t, x)-p^{\varepsilon}(t, z)\right| & \leq C|x-z|^{\sigma_{2}},
\end{aligned}
$$

for $t, s>0, x, z \in \mathbb{R}^{d}$ and some $\sigma_{1}>0$ and some $\sigma_{2}>0$.

On the other hand, as $\varepsilon$ converges to zero, the solution to (1.2) also tends to zero. So, we should expect the density $p_{t, x}^{\varepsilon}(t, x)$ to converge to a degenerate density. In this paper we will study the rate of this convergence. In particular, we will deal with the behavior of

$$
\lim _{\varepsilon \downarrow 0} \varepsilon^{2} \log p_{t, x}^{\varepsilon}(t, x) .
$$


We will use the formulation of the papers of Ben Arous, Leandre and Russo and summarized by Nualart [31] for general Wiener functionals.

All these results can also be obtained if we consider the following more general kind of stochastic partial differential equations

$$
\partial_{t} u^{\varepsilon}(t, x)+(I-\Delta)^{\frac{\alpha}{2}}(-\Delta)^{\frac{\beta}{2}} u^{\varepsilon}(t, x)=\varepsilon \theta_{1}\left(u^{\varepsilon}(t, x)\right) \dot{W}(t, x)+\theta_{2}\left(u^{\varepsilon}(t, x)\right), \quad \varepsilon>0,
$$

with $\theta_{1}, \theta_{2}: \mathbb{R} \rightarrow \mathbb{R}$. In this paper we have dealt with (1.1) for the sake of simplicity.

The paper is organized as follows. In Section 2 we will introduce the Gaussian noise and describe what we mean by a solution of (1.1). We will also present the statements of the theorems that will be proved in this paper and some results related to the calculus of this work. In Section 3 we will establish the Hölder continuity in time and in space for the density of the law of the solution. Finally, Section 4 is devoted to prove the behavior (1.3). As usually, all constants will be denoted by $C$, independently of its value.

\section{THE FRAMEWORK}

Let $\mathcal{D}\left(\mathbb{R}^{d+1}\right)$ be the space of Schwartz test functions (see, for instance, Schwartz [36] for more information). Then, the noise $W=\left\{W(\phi), \phi \in \mathcal{D}\left(\mathbb{R}^{d+1}\right)\right\}$ is an $L^{2}(\Omega, \mathcal{F}, P)$ - valued centered Gaussian process on some probability space $(\Omega, \mathcal{F}, P)$, with covariance functional

$$
J(\phi, \psi)=\int_{\mathbb{R}_{+}} \mathrm{d} s \int_{\mathbb{R}^{d}} \Gamma(\mathrm{d} s)[\phi(s, \bullet) * \tilde{\psi}(s, \bullet)](x),
$$

where $\tilde{\psi}(s, x)=\psi(s,-x)$ and $\Gamma$ is a non-negative and non-negative definite tempered measure, therefore symmetric. If we denote by $\mu$ the spectral measure of $\Gamma$, then,

$$
J(\phi, \psi)=\int_{\mathbb{R}_{+}} \mathrm{d} s \int_{\mathbb{R}^{d}} \mu(\mathrm{d} \xi) \mathcal{F} \phi(s, \bullet)(\xi) \overline{\mathcal{F} \psi(s, \bullet)(\xi)},
$$

where $\mathcal{F}$ denotes the Fourier transform and $\bar{z}$ is the complex conjugate of $z$. Since the spectral measure $\mu$ is a non-trivial and non-negative tempered measure, we can ensure that there exist positive constants $c_{1}, c_{2}, k$ such that

$$
c_{1}<\int_{\{|\xi| \leq k\}} \mu(\mathrm{d} \xi)<c_{2} .
$$

For more details on this topic, the reader is referred, for example, to the paper [7].

The Gaussian process $W$ can be extended to a worthy martingale measure, in the sense given by Walsh [38], $W=\left\{W_{t}(A), t \in \mathbb{R}_{+}, A \in \mathcal{B}_{b}\left(\mathbb{R}^{d}\right)\right\}$, where we use the same notation by the Gaussian process and its extended worthy martingale measure. In Dalang and Frangos [10] and Dalang [11], they presented an extension of Walsh's stochastic integral that requires the following integrability condition in terms of the Fourier transform of $\Gamma$ and $K$ :

$$
\int_{0}^{T} \mathrm{~d} t \int_{\mathbb{R}^{d}} \mu(\mathrm{d} \xi)\left|\mathcal{F} K_{t}(\bullet)(\xi)\right|^{2}<\infty
$$

where $K$ is the fundamental solution of (1.1). Provided that (2.2) is satisfied and assuming that $\theta$ is a Lipschitz function, we can prove that (1.2) has a solution in the sense given then and where the stochastic integral in (1.2) is defined with respect to the $\mathcal{F}_{t}$-martingale measure $W_{t}$.

On the other hand, following the ideas given in [4] or [12], the equation satisfied by the fundamental solution of (1.1) is equivalent to

$$
\partial_{t} \mathcal{F} K_{t}(\bullet)(\xi)+|\xi|^{\alpha}\left(1+|\xi|^{2}\right)^{\frac{\beta}{2}} \mathcal{F} K_{t}(\bullet)(\xi)=0
$$


which has a unique solution given by

$$
\mathcal{F} K_{t}(\bullet)(\xi)=\exp \left\{-t|\xi|^{\alpha}\left(1+|\xi|^{2}\right)^{\frac{\beta}{2}}\right\} .
$$

So, this fundamental solution can be written as

$$
K_{t}(x)=\frac{1}{(2 \pi)^{n}} \int_{\mathbb{R}^{d}} \mathrm{e}^{\mathrm{i}\langle x, \xi\rangle} \exp \left\{-t|\xi|^{\alpha}\left(1+|\xi|^{2}\right)^{\frac{\beta}{2}}\right\} \mathrm{d} \xi .
$$

In Márquez-Carreras [25], we have proved that the hypothesis on the spectral measure $\mu$

$$
\int_{\mathbb{R}^{d}} \frac{\mu(\mathrm{d} \xi)}{\left(1+|\xi|^{2}\right)^{\frac{\alpha+\beta}{2}}}<\infty
$$

implies that (2.2) is satisfied. Moreover, assuming also (2.3) and that $\theta$ is globally Lipschitz, we can prove that (1.2) has a unique adapted solution and, for any $T>0$ and $p \in[1,+\infty)$,

$$
\sup _{\varepsilon \in(0,1]} \sup _{(t, x) \in[0, T] \times \mathbb{R}^{d}} \mathbf{E}\left(\left|u^{\varepsilon}(t, x)\right|^{p}\right)<\infty,
$$

and this unique solution is mean-square continuous.

The following theorem we will be the first important result of this paper. It will be proved in the next section.

Theorem 2.1. Suppose that the coefficient satisfies:

(H1) The function $\theta$ is $\mathcal{C}^{\infty}$ with bounded derivatives of any order.

(H2) There exists $\theta_{0}>0$ such that $|\theta(z)|>\theta_{0}$ for any $z \in \mathbb{R}$.

Assume also that the spectral mesure $\mu$ associated to $W$ satisfies

$$
\int_{\mathbb{R}^{d}} \frac{\mu(\mathrm{d} \xi)}{\left(1+|\xi|^{2}\right)^{\frac{q(\alpha+\beta)}{2}}}<\infty
$$

for some $q \in\left(0, \frac{1}{2}\right)$. Then, the law of $u(t, x)$, solution to (1.2) for $\varepsilon=1$, is absolutely continuous with respecte to Lebesgue's measure on $\mathbb{R}$ and its density $p_{t, x}(t, x)$ is infinitely differentiable. Moreover, for every $s, t \in[0, T]$, $T>0, x, z \in \mathbb{R}^{d}, \rho_{1} \in\left(0, \frac{1-q}{2}\right)$ and $\rho_{2} \in(0,1-q)$, we have that

$$
\begin{aligned}
& \left|p_{t, x}(y)-p_{s, x}(y)\right| \leq C|t-s|^{\rho_{1}}, \\
& \left|p_{t, x}(y)-p_{t, z}(y)\right| \leq \begin{cases}C|z-x|^{\rho_{2}}, & \text { if } \alpha+\beta \geq 2, \\
C|z-x|^{\frac{\rho_{2}(\alpha+\beta)}{2}}, & \text { if } \alpha+\beta<2 .\end{cases}
\end{aligned}
$$

Remark 2.2. In the stochastic heat equation, a more particular case, the authors considered the same assumptions on the coefficients, (H1) and (H2).

Let $\mathcal{E}$ be the space of measurables functions $\phi: \mathbb{R}^{d} \rightarrow \mathbb{R}^{d}$ such that

$$
\int_{\mathbb{R}^{d}} \Gamma(\mathrm{d} s)[\phi(\bullet) * \tilde{\psi}(\bullet)](x)<\infty .
$$

This space $\mathcal{E}$ is endowed with a natural inner product $\langle\phi, \psi\rangle_{\mathcal{E}}$. Let $\mathcal{H}$ be the completation of $\mathcal{E}$ and $\mathcal{H}_{T}=$ $L^{2}([0, T], \mathcal{H}) . \mathcal{H}_{T}$ is a real separable Hilbert space such that, if $\phi, \psi \in \mathcal{D}\left([0, T] \times \mathbb{R}^{d}\right)$,

$$
\mathbf{E}[W(\phi) W(\psi)]=\langle\phi, \psi\rangle_{\mathcal{H}_{T}} .
$$


For $x=\left(x_{1}, \ldots, x_{n}\right) \in \mathbb{R}^{d}$ and $a \in \mathbb{R}$, we denote by $R(x)$ the minimum rectangle containing 0 and $x$ and by $\operatorname{sgn}(a)$ the sign of $a$. The space $H$ will be the set of functions $\bar{h}$ such that, for any $(t, x) \in \mathbb{R}_{+} \times \mathbb{R}^{d}$ and $h \in \mathcal{H}_{T}$, we have that

$$
\bar{h}(t, x)=\operatorname{sgn}\left(x_{1} \times \cdots \times x_{n}\right)\left\langle\mathbb{1}_{[0, T] \times R(x)}, h\right\rangle_{\mathcal{H}_{T}},
$$

with the scalar product $\langle\bar{h}, \bar{k}\rangle_{H}=\langle h, k\rangle_{\mathcal{H}_{T}} . H$ is a Hilbert space isomorphic to $\mathcal{H}_{T}$.

For any $\bar{h} \in H$, we consider the deterministic evolution equation

$$
\Psi^{\bar{h}}(t, x)=\left\langle K_{t-.}(x-\bullet) \theta\left(\Psi^{\bar{h}}(\cdot, \bullet)\right), h\right\rangle_{\mathcal{H}_{T}}, \quad \forall(t, x) \in \mathbb{R}_{+} \times \mathbb{R}^{d} .
$$

Finally, we will be able to state the second main result that will be proved in Section 4 .

Theorem 2.3. Assume the hypothesis on the coefficient (H1) and (H2) and the assumption on the spectral measure (2.4) for some $q \in\left(0, \frac{1}{2}\right)$. Then, the density of the law of $u^{\varepsilon}(t, x)$ has the following behavior

$$
\lim _{\varepsilon \downarrow 0} \varepsilon^{2} \log p_{t, x}^{\varepsilon}(t, x)=-d_{t}^{2}(x, y),
$$

with

$$
d_{t}^{2}(x, y)=\inf \left\{\frac{1}{2}\|\bar{h}\|_{H}^{2}, \Psi^{\bar{h}}(t, x)=y\right\}
$$

\section{The EXISTEnCE OF A SMOOTH DENSITY}

The main but of this section is to prove Theorem 2.1. We first introduce some notations and quote notions that will be used throughout this section and the paper.

The centered Gaussian noise $W$ can be identified with a Gaussian process $\left\{B(h), h \in \mathcal{H}_{T}\right\}$ as follows. Let $\left\{e_{j} ; j \geq 0\right\} \subset \mathcal{E}$ be a CONS of the Hilbert space $\mathcal{H}$, then

$$
B_{j}(t)=\int_{0}^{t} \int_{\mathbb{R}^{d}} e_{j}(x) W(\mathrm{~d} s, \mathrm{~d} x), \quad j \in \mathbb{N}, t \in[0, T]
$$

is a sequence of independent standard Brownion motions such that

$$
W(\phi)=\sum_{j=0}^{\infty} \int_{0}^{T}\left\langle\phi(s, \bullet), e_{j}(\bullet)\right\rangle_{\mathcal{H}} d B_{j}(s), \quad \phi \in \mathcal{D}\left([0, T] \times \mathbb{R}^{d}\right) .
$$

For $h \in \mathcal{H}_{T}$, we set

$$
B(h)=\sum_{j=0}^{\infty} \int_{0}^{T}\left\langle h(s), e_{j}\right\rangle_{\mathcal{H}} d B_{j}(s) .
$$

Then, we can use the framework of the Malliavin calculus developed in Nualart [31] (see also Nualart [32]). The Sobolev spaces $\mathbb{D}^{l, p}$ are defined by means of iterations of the derivative operator $D$. For a random variable $X$, $D^{l} X$ defines a $\mathcal{H}_{T}^{\otimes l}$-valued random variable if it exists. For $h \in \mathcal{H}_{T}$, set $D_{h} X=\langle D X, h\rangle_{\mathcal{H}_{T}}$ and for $r \in[0, T]$, $D_{r} X$ defines an element of $\mathcal{H}$, which is denoted by $D_{r, \bullet} X$. Then, for any $h \in \mathcal{H}_{T}$,

$$
D_{h} X=\int_{0}^{T}\left\langle D_{r, \bullet} X, h(r)\right\rangle_{\mathcal{H}} \mathrm{d} r
$$

We write $D_{r, \phi} X=\left\langle D_{r, \bullet} X, \phi\right\rangle_{\mathcal{H}}$ for $\phi \in \mathcal{H}$.

It is no difficult to prove the following result. 
Proposition 3.1. Assume (H1) and (2.3). Then, for any $t \geq 0, x \in \mathbb{R}^{d}, \varepsilon>0$, we have that the solution $u^{\varepsilon}(t, x)$ to (1.2) belongs to $\mathbb{D}^{\infty}=\cap_{p \geq 1} \cap_{l \geq 1} \mathbb{D}^{l, p}(\mathbb{R})$. Moreover, for any $l \geq 1, p \in[1, \infty)$

$$
\sup _{\varepsilon \in(0,1]}\left\|u^{\varepsilon}(t, x)\right\|_{l, p}<\infty .
$$

Proof. See Theorem 2.1 in [26].

Moreover, the derivative satisfies, for any $\phi \in \mathcal{H}$.

$$
\begin{aligned}
D_{r, \phi} u^{\varepsilon}(t, x)= & \varepsilon\left\langle K_{t-r}(x-\bullet) \theta\left(u^{\varepsilon}(r, \bullet), \phi\right\rangle_{\mathcal{H}}\right. \\
& +\varepsilon \int_{r}^{t} \int_{\mathbb{R}^{d}} K_{t-s}(x-y) D_{r, \phi} u^{\varepsilon}(s, y) \theta^{\prime}\left(u^{\varepsilon}(s, y)\right) W(\mathrm{~d} s, \mathrm{~d} y),
\end{aligned}
$$

if $r \in[0, t]$, and $D_{r, \phi} u^{\varepsilon}(t, x)=0$ if $r>t$.

To deal with the greater order Malliavin derivatives we would borrow the notations of Millet and SanzSolé [30].

We will need the following lemma proved by induction (see, for instance, [24]). It follows from the duality between the Malliavin derivative and the Skorohod integral operator $\delta$.

Lemma 3.2. Let $X, Z \in \mathbb{D}^{\infty}$ satisfying $\langle D Z, D Z\rangle_{\mathcal{H}_{T}}^{-1} \in \cap_{p \geq 1} L^{p}(\Omega)$. Define $H_{0}(X, Z)=X$ and

$$
H_{n+1}(X, Z)=\delta\left(H_{n}(X, Z), \frac{D Z}{\langle D Z, D Z\rangle_{\mathcal{H}_{T}}}\right), \quad n \geq 0 .
$$

Then, for any integers $n, l \geq 1$ and $p \in[1, \infty)$,

$$
\left\|H_{n}(X, Z)\right\|_{l, p} \leq C\|X\|_{l+n, 4^{n} p},
$$

where $C$ is a constant depending on the following norms:

$$
\|Z\|_{l+2,4^{n} p} \quad \text { and } \quad \mathbf{E}\left[\langle D Z, D Z\rangle_{\mathcal{H}_{T}}^{-1}\right]^{l(n)} \quad \text { with } l(n) \in \mathbb{N} .
$$

We now have all the requirements to prove Theorem 2.1.

Proof of Theorem 2.1. In Theorem 4.1 of Márquez-Carreras [25] we established that the law of $u(t, x)$ is absolutely continuous with respect to Lebesgue's measure on $\mathbb{R}$ and its density is infinitely differentiable.

Now we prove the second result. Let $f$ be a smooth real function. Denote by $F$ its primitive. We next check, for any $\tau>0$ such that $t, t+\tau \in[0, T]$, and $x, \eta \in \mathbb{R}^{d}$,

$$
\begin{aligned}
& |\mathbf{E}[f(u(t+\tau, x))-f(u(t, x))]| \leq C\|F\|_{\infty}|\tau|^{\rho_{1},} \\
& |\mathbf{E}[f(u(t, x+\eta))-f(u(t, x))]| \leq \begin{cases}C\|F\|_{\infty}|\eta|^{\rho_{2}}, & \text { if } \alpha+\beta \geq 2, \\
C\|F\|_{\infty}|\eta|^{\frac{\rho_{2}(\alpha+\beta)}{2}}, & \text { if } \alpha+\beta<2 .\end{cases}
\end{aligned}
$$

The case $\tau<0$ is analogous. We first show (3.2). Consider the Taylor expansion up to second order

$$
\mathbf{E}[f(u(t+\tau, x))-f(u(t, x))]=M_{t, x}(\tau)+N_{t, x}(\tau),
$$

with

$$
\begin{aligned}
M_{t, x}(\tau) & =\mathbf{E}\left[f^{\prime}(u(t, x))(u(t+\tau, x)-u(t, x))\right], \\
N_{t, x}(\tau) & =\mathbf{E}\left[(u(t+\tau, x)-u(t, x))^{2} \int_{0}^{1}(1-\lambda) f^{\prime \prime}\left(\Lambda_{t, x}(\tau, \lambda)\right) \mathrm{d} \lambda\right],
\end{aligned}
$$


and

$$
\Lambda_{t, x}(\tau, \lambda)=u(t, x)+\lambda(u(t+\tau, x)-u(t, x)) .
$$

We can decompose $M$ into two terms as follows:

$$
M_{t, x}(\tau)=M_{t, x}^{1}(\tau)+M_{t, x}^{2}(\tau)
$$

with

$$
\begin{aligned}
& M_{t, x}^{1}(\tau)=\mathbf{E}\left[f^{\prime}(u(t, x)) \int_{0}^{t} \int_{\mathbb{R}^{d}}\left(K_{t+\tau-s}(x-y)-K_{t-s}(x-y)\right) \theta(u(s, y)) W(\mathrm{~d} s, \mathrm{~d} y)\right], \\
& M_{t, x}^{2}(\tau)=\mathbf{E}\left[f^{\prime}(u(t, x)) \int_{t}^{t+\tau} \int_{\mathbb{R}^{d}} K_{t+\tau-s}(x-y) \theta(u(s, y)) W(\mathrm{~d} s, \mathrm{~d} y)\right] .
\end{aligned}
$$

Let

$$
\tilde{M}_{t, x}^{1}(\tau)=\int_{0}^{t} \int_{\mathbb{R}^{d}}\left(K_{t+\tau-s}(x-y)-K_{t-s}(x-y)\right) \theta(u(s, y)) W(\mathrm{~d} s, \mathrm{~d} y) .
$$

Applying repeatedly the integration by parts formula and Lemma 3.2 we obtain

$$
\begin{aligned}
\left|M_{t, x}^{1}(\tau)\right| & =\left|\mathbf{E}\left[F(u(t, x)) H_{2}\left(\tilde{M}_{t, x}^{1}(\tau), u(t, x)\right)\right]\right| \\
& \leq\|F\|_{\infty} \mathbf{E}\left[\left|H_{2}\left(\tilde{M}_{t, x}^{1}(\tau), u(t, x)\right)\right|\right] \\
& \leq C\|F\|_{\infty}\left\|\tilde{M}_{t, x}^{1}(\tau)\right\|_{2,16} \\
& =C\|F\|_{\infty}\left(\mathbf{E}\left[\left|\tilde{M}_{t, x}^{1}(\tau)\right|^{16}\right]+\sum_{i=1}^{2} \mathbf{E}\left[\left\|D^{i} \tilde{M}_{t, x}^{1}(\tau)\right\|_{\mathcal{H}_{T}^{\otimes i}}^{16}\right]\right)^{\frac{1}{16}} .
\end{aligned}
$$

First, Burkholder's inequality, the assumption on $\theta$ and relation between $\Gamma$ and $\mu$ imply that

$$
\mathbf{E}\left[\left|\tilde{M}_{t, x}^{1}(\tau)\right|^{16}\right] \leq\left[\int_{0}^{t} \int_{\mathbb{R}^{d}} \mu(\mathrm{d} \xi)\left(\mathcal{F} K_{t+\tau-s}(\bullet)(\xi)-\mathcal{F} K_{t-s}(\bullet)(\xi)\right)^{2}\right]^{8}=M_{t, x}^{1,1}(\tau)+M_{t, x}^{1,2}(\tau),
$$

with

$$
\begin{aligned}
& M_{t, x}^{1,1}(\tau)=\left[\int_{0}^{t} \int_{\{|\xi|<k\}} \mu(\mathrm{d} \xi) \mathrm{e}^{-2(t-s)|\xi|^{\alpha}\left(1+|\xi|^{2}\right)^{\frac{\beta}{2}}}\left(1-\mathrm{e}^{-\tau|\xi|^{\alpha}\left(1+|\xi|^{2}\right)^{\frac{\beta}{2}}}\right)^{2}\right]^{8} \\
& M_{t, x}^{1,2}(\tau)=\left[\int_{0}^{t} \mathrm{~d} s \int_{\{|\xi| \geq k\}} \mu(\mathrm{d} \xi) \mathrm{e}^{-2(t-s)|\xi|^{\alpha}\left(1+|\xi|^{2}\right)^{\frac{\beta}{2}}}\left(1-\mathrm{e}^{-\tau|\xi|^{\alpha}\left(1+|\xi|^{2}\right)^{\frac{\beta}{2}}}\right)^{2}\right]^{8} .
\end{aligned}
$$

Let $\rho_{1} \in(0,(1-q) / 2)$. Fubini's theorem, the facts that $1-\mathrm{e}^{-x} \leq 1$ and $1-\mathrm{e}^{-x} \leq x, \forall x>0$, and the estimate (2.4) yield that

$$
\begin{aligned}
M_{t, x}^{1,2}(\tau) & \leq C\left[\int_{\{|\xi| \geq k\}} \frac{1-\exp \left\{-2 \tau|\xi|^{\alpha}\left(1+|\xi|^{2}\right)^{\frac{\beta}{2}}\right\}}{|\xi|^{\alpha}\left(1+|\xi|^{2}\right)^{\frac{\beta}{2}}} \mu(\mathrm{d} \xi)\right]^{8} \\
& \leq C\left[\int_{\{|\xi| \geq k\}} \frac{\left[1-\exp \left\{-2 \tau|\xi|^{\alpha}\left(1+|\xi|^{2}\right)^{\frac{\beta}{2}}\right\}\right]^{2 \rho_{1}}}{|\xi|^{\alpha}\left(1+|\xi|^{2}\right)^{\frac{\beta}{2}}} \mu(\mathrm{d} \xi)\right]^{8} \\
& \leq C|\tau|^{16 \rho_{1}}\left[\int_{\{|\xi| \geq k\}} \frac{\mu(\mathrm{d} \xi)}{\left(1+|\xi|^{2}\right)^{\frac{\left(1-2 \rho_{1}\right)(\alpha+\beta)}{2}}}\right]^{8} \\
& \leq C|\tau|^{16 \rho_{1}} .
\end{aligned}
$$


By means of (2.1) we can easily get the following bound:

$$
M_{t, x}^{1,1}(\tau) \leq C|\tau|^{p} .
$$

We also have to study the first and the second Malliavin derivatives of $\tilde{M}_{t, x}^{1}(\tau)$. We deal with the first one

$$
\begin{aligned}
\mathbf{E}\left[\left\|D \tilde{M}_{t, x}^{1}(\tau)\right\|_{\mathcal{H}_{T}}^{16}\right] \leq C\left(\mathbf{E}\left[\left\|\left(K_{t+\tau-.}(x-\bullet)-K_{t-.}(x-\bullet)\right) \theta(u(\cdot, \bullet))\right\|_{\mathcal{H}_{T}}^{16}\right]\right. \\
\left.\quad+\mathbf{E}\left[\left\|\int_{0}^{t} \int_{\mathbb{R}^{d}}\left(K_{t+\tau-s}(x-y)-K_{t-s}(x-y)\right) \theta^{\prime}(u(s, y)) D u(s, y) W(\mathrm{~d} s, \mathrm{~d} y)\right\|_{\mathcal{H}_{T}}^{16}\right]\right) .
\end{aligned}
$$

The first term of the right side of inequality is bounded as in (3.4). In order to study the second one we have to take into account that, for any $\gamma \in[0, t]$,

$$
Z_{\gamma}=\int_{0}^{\gamma} \int_{\mathbb{R}^{d}}\left(K_{t+\tau-s}(x-y)-K_{t-s}(x-y)\right) \theta^{\prime}(u(s, y)) D u(s, y) W(\mathrm{~d} s, \mathrm{~d} y)
$$

is a continuous $\mathcal{H}_{T}$-valued $\mathcal{F}_{\gamma}$-martingale and that one can check that

$$
\langle Z\rangle_{\gamma}=\sum_{j \geq 0}\left\|1_{[0, \gamma]}\left(K_{t+\tau-} .(x-\bullet)-K_{t-.}(x-\bullet)\right) \theta^{\prime}(u(\cdot, \bullet)) D_{e_{j}} u(\cdot, \bullet)\right\|_{\mathcal{H}_{T}}^{2}
$$

is the unique predictable increasing process such that $\langle Z\rangle_{0}=0$ and $\left\|Z_{\gamma}\right\|_{\mathcal{H}_{T}}^{2}-\langle Z\rangle_{\gamma}$ is a real $\mathcal{F}_{\gamma}$-martingale and $\left\{e_{j}, j \geq 0\right\}$ is a CONS of the Hilbert space $\mathcal{H}$. Then, Burkholder's inequality for a $\mathcal{H}_{T}$-valued martingale, Parseval's identity and Schwartz's inequality applied to the scalar product in $\mathcal{H}_{T}$ yield that the second term in the right side of (3.5) is bounded by

$$
\begin{aligned}
& C \mathbf{E} \mid \sum_{j \geq 0} \int_{0}^{t} \mathrm{~d} s \int_{\mathbb{R}^{d}} \Gamma(\mathrm{d} y) \int_{\mathbb{R}^{d}} \mathrm{~d} y^{\prime}\left[K_{t+\tau-s}\left(x-y+y^{\prime}\right)-K_{t-s}\left(x-y+y^{\prime}\right)\right] \theta^{\prime}\left(u\left(s, y-y^{\prime}\right)\right) \\
& \quad \times\left. D_{e_{j}} u\left(s, y-y^{\prime}\right)\left[K_{t+\tau-s}\left(x+y^{\prime}\right)-K_{t-s}\left(x+y^{\prime}\right)\right] \theta^{\prime}\left(u\left(s, y^{\prime}\right)\right) D_{e_{j}} u\left(s, y^{\prime}\right)\right|^{8} \\
& \leq C \mathbf{E} \mid \int_{0}^{t} \mathrm{~d} s \int_{\mathbb{R}^{d}} \Gamma(\mathrm{d} y) \int_{\mathbb{R}^{d}} \mathrm{~d} y^{\prime}\left[K_{t+\tau-s}\left(x-y+y^{\prime}\right)-K_{t-s}\left(x-y+y^{\prime}\right)\right] \theta^{\prime}\left(u\left(s, y-y^{\prime}\right)\right) \\
& \quad \times\left.\left\langle D u\left(s, y-y^{\prime}\right), D u\left(s, y^{\prime}\right)\right\rangle_{\mathcal{H}_{T}}\left[K_{t+\tau-s}\left(x+y^{\prime}\right)-K_{t-s}\left(x+y^{\prime}\right)\right] \theta^{\prime}\left(u\left(s, y^{\prime}\right)\right)\right|^{8} .
\end{aligned}
$$

Now, Hölder's inequality, the bounded condition on $\theta^{\prime}$ and an argument similar to (3.4) give the desired result. Not very different calculus allow to work with the second Malliavin derivative of $\tilde{M}_{t, x}^{1}(\tau)$. It would finish the study of $M_{t, x}^{1}(\tau)$.

We now analize $M_{t, x}^{2}(\tau)$. The variable $f^{\prime}(u(t, x))$ is $\mathcal{F}_{t}$-measurable and

$$
\int_{0}^{\gamma} \int_{\mathbb{R}^{d}} K_{t+\tau-s}(x-y) \theta(u(s, y)) W(\mathrm{~d} s, \mathrm{~d} y)
$$

is an $L^{2}$-bounded $\mathcal{F}_{\gamma}$-martingale. Then, by independence it is clear that $M_{t, x}^{2}(\tau)=0$. It concludes the study of $M$.

In order to prove (3.2) it only remains to deal with $N$. By means of Lemma 3.2 we have that

$$
\left|N_{t, x}(\tau)\right| \leq C\|F\|_{\infty}\left\|(u(t+\tau, x)-u(t, x))^{2}\right\|_{3,64} .
$$


It is not difficult to check that

$$
\left\|(u(t+\tau, x)-u(t, x))^{2}\right\|_{n, p} \leq C|\tau|^{\rho_{1}} .
$$

The case $n=0$ is as in Proposition 4.1 of [25]. For $n \geq 1$, this inequality can be proved as before taking into account that $u(t, x) \in \mathbb{D}^{\infty}$ for any $(t, x) \in[0, T] \times \mathbb{R}^{d}$ and that the difference in the last norm is to square.

So, we have that

$$
|\mathbf{E}[f(u(t+\tau, x))-f(u(t, x))]| \leq C|| F \|_{\infty}|\tau|^{\rho_{1}} .
$$

Fix $y \in \mathbb{R}$ and consider the Dirac delta function $\delta_{\{y\}}$. Let $\left\{f_{n}, n \geq 1\right\}$ be a sequence of smooth functions converging to $\delta_{\{y\}}$. By passing to the limit the term of the left side in the last inequality for $n \rightarrow \infty$, we obtain (2.5) in Theorem 2.1.

The proof of the Hölder continuity with respect to the space is analogous. We will only give some important steps of this proof. First of all, the Taylor expansion up to second order implies that

$$
\mathbf{E}[f(u(t, x+\eta))-f(u(t, x))]=O_{t, x}(\eta)+R_{t, x}(\eta),
$$

with

$$
O_{t, x}(\eta)=\mathbf{E}\left[f^{\prime}(u(t, x))(u(t, x+\eta)-u(t, x))\right],
$$

and $R_{t, x}(\eta)$ defined in a similar way to the previous case $N_{t, x}(\tau)$.

So, by integration by parts formula we have

$$
O_{t, x}(\eta)=\mathbf{E}\left[f^{\prime}(u(t, x)) \tilde{O}_{t, x}(\eta)\right]=\mathbf{E}\left[F(u(t, x)) H_{2}\left(\tilde{O}_{t, x}(\eta), u(t, x)\right)\right],
$$

with

$$
\tilde{O}_{t, x}(\eta)=\int_{0}^{t} \int_{\mathbb{R}^{d}}\left[K_{t-s}(x+\eta-y)-K_{t-s}(x-y)\right] \theta(u(s, y)) W(\mathrm{~d} s, \mathrm{~d} y) .
$$

Then, Lemma 3.2 ensures that

$$
\left|O_{t, x}(\eta)\right| \leq C\|F\|_{\infty}\left\|\tilde{O}_{t, x}(\eta)\right\|_{2,16} .
$$

We bound the expectation of $\tilde{O}_{t, x}(\eta)$ in order to ilustrate the reader as we deal with this case. Burkholder's inequality yields

$$
\begin{aligned}
& \mathbf{E}\left[\left|\int_{0}^{t} \int_{\mathbb{R}^{d}}\left[K_{t-s}(x+\eta-y)-K_{t-s}(x-y)\right] \theta(u(s, y)) W(\mathrm{~d} s, \mathrm{~d} y)\right|^{16}\right] \\
& \quad \leq C\left[\int_{0}^{t} \mathrm{~d} s \int_{\mathbb{R}^{d}} \mu(\mathrm{d} \xi)\left|\mathcal{F} K_{t-s}(x+\eta-\bullet)(\xi)-\mathcal{F} K_{t-s}(x-\bullet)(\xi)\right|^{2}\right]^{8} \\
& \quad \leq C\left[O_{t, x}^{1}(\eta)+O_{t, x}^{2}(\eta)\right],
\end{aligned}
$$

with

$$
\begin{aligned}
& O_{t, x}^{1}(\eta)=\left[\int_{0}^{t} \mathrm{~d} s \int_{\{|\xi| \leq k\}} \mu(\mathrm{d} \xi)\left|\mathcal{F} K_{t-s}(x+\eta-\bullet)(\xi)-\mathcal{F} K_{t-s}(x-\bullet)(\xi)\right|^{2}\right]^{8}, \\
& O_{t, x}^{2}(\eta)=\left[\int_{0}^{t} \mathrm{~d} s \int_{\{|\xi|>k\}} \mu(\mathrm{d} \xi)\left|\mathcal{F} K_{t-s}(x+\eta-\bullet)(\xi)-\mathcal{F} K_{t-s}(x-\bullet)(\xi)\right|^{2}\right]^{8} .
\end{aligned}
$$


It is easy to deal with $O_{t, x}^{1}(\eta)$. The bound will not depend on the value of $\alpha$ and $\beta$. The fact that the Fourier transform of $K$ is bounded by 1 , the mean value theorem and the property (2.1) imply that

$$
\begin{aligned}
O_{t, x}^{1}(\eta) & =\left[\int_{0}^{t} \mathrm{~d} s \int_{\{|\xi| \leq k\}} \mu(\mathrm{d} \xi)\left|\mathrm{e}^{-\mathrm{i}\langle x+\eta, \xi\rangle}-\mathrm{e}^{-\mathrm{i}\langle x, \xi\rangle}\right|^{2}\left|\mathcal{F} K_{t-s}(\bullet)(\xi)\right|^{2}\right]^{8} \\
& \leq C\left[\int_{0}^{t} \mathrm{~d} s \int_{\{|\xi| \leq k\}} \mu(\mathrm{d} \xi)|\langle\eta, \xi\rangle|^{2}\right]^{8} \\
& \leq C|\eta|^{16} .
\end{aligned}
$$

We analyze the other term. Recall that $\rho_{2} \in(0,1-q)$. Here we will distinguish two cases depending on the value of $\alpha$ and $\beta$. We first consider the case $\alpha+\beta \geq 2$. Using the mean value theorem, Fubini's theorem, the fact that $1-\mathrm{e}^{-x} \leq 1$ for all $x>0$, and (2.4), we obtain

$$
\begin{aligned}
O_{t, x}^{2}(\eta) & =4\left[\int_{0}^{t} \mathrm{~d} s \int_{\{|\xi|>k\}} \mu(\mathrm{d} \xi)\left|\frac{\mathrm{e}^{-\mathrm{i}\langle x+\eta, \xi\rangle}-\mathrm{e}^{-\mathrm{i}\langle x, \xi\rangle}}{2}\right|^{2}\left|\mathcal{F} K_{t-s}(\bullet)(\xi)\right|^{2}\right]^{8} \\
& \leq 4\left[\int_{0}^{t} \mathrm{~d} s \int_{\{|\xi|>k\}} \mu(\mathrm{d} \xi)\left|\frac{\mathrm{e}^{-\mathrm{i}\langle x+\eta, \xi\rangle}-\mathrm{e}^{-\mathrm{i}\langle x, \xi\rangle}}{2}\right|^{2 \rho_{2}}\left|\mathcal{F} K_{t-s}(\bullet)(\xi)\right|^{2}\right]^{8} \\
& \leq C\left[\int_{0}^{t} \mathrm{~d} s \int_{\{|\xi|>k\}} \mu(\mathrm{d} \xi)|\eta|^{2 \rho_{2}}|\xi|^{2 \rho_{2}} \exp \left\{-2(t-s)|\xi|^{\alpha}\left(1+|\xi|^{2}\right)^{\frac{\beta}{2}}\right\}\right]^{8} \\
& \left.\leq C|\eta|^{16 \rho_{2}}\left[\int_{\{|\xi|>k\}} \mu(\mathrm{d} \xi) \frac{|\xi|^{2 \rho_{2}}}{|\xi|^{\alpha}\left(1+|\xi|^{2}\right)^{\frac{\beta}{2}}}\right]^{8}\right]^{8} \\
& \leq C|\eta|^{16 \rho_{2}}\left[\int_{\{|\xi|>k\}} \mu(\mathrm{d} \xi) \frac{1}{\left(1+|\xi|^{2}\right)^{\frac{\left(1-\rho_{2}\right)(\alpha+\beta)}{2}}}\right]^{\leq} \\
& \leq C|\eta|^{16 \rho_{2}} .
\end{aligned}
$$

The case $\alpha+\beta<2$ is bounded in a similar way as follows:

$$
\begin{aligned}
O_{t, x}^{2}(\eta) & \leq 4\left[\int_{0}^{t} \mathrm{~d} s \int_{\{|\xi|>k\}} \mu(\mathrm{d} \xi)\left|\frac{\mathrm{e}^{-\mathrm{i}\langle x+\eta, \xi\rangle}-\mathrm{e}^{-\mathrm{i}\langle x, \xi\rangle}}{2}\right|^{\rho_{2}(\alpha+\beta)}\left|\mathcal{F} K_{t-s}(\bullet)(\xi)\right|^{2}\right]^{8} \\
& \leq C|\eta|^{8 \rho_{2}(\alpha+\beta)}\left[\int_{\{|\xi|>k\}} \mu(\mathrm{d} \xi) \frac{|\xi|^{\rho_{2}(\alpha+\beta)}}{|\xi|^{\alpha}\left(1+|\xi|^{2}\right)^{\frac{\beta}{2}}}\right]^{8} \\
& \leq C|\eta|^{8 \rho_{2}(\alpha+\beta)}\left[\int_{\{|\xi|>k\}} \mu(\mathrm{d} \xi) \frac{1}{\left(1+|\xi|^{2}\right)^{\frac{\left(1-\rho_{2}\right)(\alpha+\beta)}{2}}}\right]^{8} \\
& \leq C|\eta|^{8 \rho_{2}(\alpha+\beta)} .
\end{aligned}
$$

The other bounds of $\tilde{O}_{t, x}(\eta)$ (the first and second Malliavin derivatives) and the study of $R_{t, x}(\eta)$ can be obtained following similar steps to the Hölder continuity with respect to the time. It would imply (3.3). Then, using the same argument as before, we would obtain (2.6), concluding the Proof of Theorem 2.1. 


\section{The STUdy of SMALl PERTURBations}

The purpose of this section is to prove Theorem 2.3. In order to obtain it we will use the formulation of the method presented by Ben Arous, Leandre and Russo and summarized by Nualart [31] for general Wiener functionals.

We will need the following ingredients. A random vector $X: \Omega \rightarrow \mathbb{R}^{d}$ is said to be non-degenerate if $X \in \mathbb{D}^{\infty}$ and the Malliavin derivative $\gamma_{X}=\langle D X, D X\rangle$ satisfies that $\operatorname{det} \gamma_{X}^{-1} \in \cap_{p \geq 1} L^{p}(\Omega)$.

Proposition 4.1 (Prop. 4.4.2 of [31]). Let $\left\{X^{\varepsilon}, \varepsilon \in(0,1]\right\}$ be a family of non-degenerate random variables satisfying

(i) $\sup _{\varepsilon \in(0,1]}\left\|X^{\varepsilon}\right\|_{l, p}<\infty$, for each integer $l \geq 1, p \in[1, \infty)$.

(ii) For any $p \in[1, \infty)$, there exists $N(p) \in[1, \infty)$ such that $\left\|\gamma_{X^{\varepsilon}}^{-1}\right\|_{p} \leq \varepsilon^{-N(p)}$.

(iii) $\left\{X^{\varepsilon}, \varepsilon \in(0,1]\right\}$ obeys a large deviation principle on $\mathbb{R}$ with rate function $L(y), y \in \mathbb{R}$.

Then, if $p^{\varepsilon}$ denotes the density of $X^{\varepsilon}$,

$$
\limsup _{\varepsilon \downarrow 0} \varepsilon^{2} \log p^{\varepsilon}(y) \leq-L(y) .
$$

Proposition 4.2 (Prop. 4.4.1 of [31]). Let $\left\{X^{\varepsilon}, \varepsilon \in(0,1]\right\}$ be a family of non-degenerate random variables. Let $\Lambda \in \mathcal{C}^{1}(H ; \mathbb{R})$ such that for each $\bar{h} \in H$

$$
\lim _{\varepsilon \downarrow 0} \frac{X^{\varepsilon}\left(\omega+\frac{\bar{h}}{\varepsilon}\right)-\Lambda(\bar{h})}{\varepsilon}=Z(\bar{h}),
$$

in the topology $\mathbb{D}^{\infty}$, where $Z(\bar{h})$, is a random variable belonging to the first Wiener Chaos with variance $\gamma_{\Lambda}(\bar{h})$ $\left(\gamma_{\Lambda}\right.$ the deterministic Malliavin matrix). Define

$$
d_{R}^{2}(y)=\inf \left\{\|\bar{h}\|_{H}^{2}, \Lambda(\bar{h})=y, \gamma_{\Lambda}(\bar{h})>0\right\} .
$$

Then, if $p^{\varepsilon}$ denotes the density of $X^{\varepsilon}$,

$$
\liminf _{\varepsilon \downarrow 0} 2 \varepsilon^{2} \log p^{\varepsilon}(y) \geq-d_{R}^{2}(y) .
$$

In next lemmas we will prove all the assumptions of these two propositions for the solution of our stochastic partial differential equation.

Lemma 4.3. Assume (H1) and (2.3). For any integer $l \geq 0$ and $p \in[1,+\infty)$, we have that

$$
\sup _{\varepsilon \in(0,1]} \sup _{(t, x) \in[0, T] \times \mathbb{R}^{d}}\left\|u^{\varepsilon}(t, x)\right\|_{l, p}<\infty .
$$

Proof. The case $l=0$ can be proved by the standard argument based on Picard's iterations:

$$
\begin{aligned}
u_{0}^{\varepsilon}(t, x) & =0, \\
u_{n}^{\varepsilon}(t, x) & =\varepsilon \int_{0}^{t} \int_{\mathbb{R}^{d}} K_{t-s}(x-y) \theta\left(u_{n-1}^{\varepsilon}(s, y)\right) W(\mathrm{~d} s, \mathrm{~d} y), \quad n \geq 1 .
\end{aligned}
$$

We easily prove that $u_{n}^{\varepsilon}(t, x)$ is well-defined and then, using Burkholder's inequality, we can check that, for any $n \geq 0$,

$$
\sup _{\varepsilon \in(0,1]} \sup _{(t, x) \in[0, T] \times \mathbb{R}^{d}} \mathbf{E}\left[\left|u_{n}^{\varepsilon}(t, x)\right|^{2}\right]<\infty,
$$


and by means of Gronwall's Lemma that

$$
\sup _{n \geq 0} \sup _{\varepsilon \in(0,1]} \sup _{(t, x) \in[0, T] \times \mathbb{R}^{d}} \mathbf{E}\left[\left|u_{n}^{\varepsilon}(t, x)\right|^{2}\right]<\infty .
$$

The same kind of arguments allows us to show these two bounds changing the power 2 for $p>2$. Morover, we can also prove that $\left\{u_{n}^{\varepsilon}(t, x), n \geq 0\right\}$ converges uniformly in $L^{p}$, denoting by $u^{\varepsilon}(t, x)$ this limit. Then, we can check that this limit is unique and

$$
\sup _{\varepsilon \in(0,1]} \sup _{(t, x) \in[0, T] \times \mathbb{R}^{d}} \mathbf{E}\left[\left|u^{\varepsilon}(t, x)\right|^{p}\right]<\infty .
$$

We have (4.1) for $l=0$. For $l \geq 1$, it can be shown following exactly the same arguments as in Proposition 2.4 of $[26]$.

Lemma 4.4. Assume (H1), (H2) and (2.4) for some $q \in\left(0, \frac{1}{2}\right)$. Then, fixed $t>0$ and $x \in \mathbb{R}^{d}$, for any $p \geq 1$, there exists a positive constant $C_{p}$ such that

$$
\left\|\left\langle D u^{\varepsilon}(t, x), D u^{\varepsilon}(t, x)\right\rangle_{\mathcal{H}_{T}}^{-1}\right\|_{p} \leq C_{p} \varepsilon^{-2}, \quad \forall \varepsilon \in(0,1]
$$

Proof. We only need to check that, for any $p \geq 2$, there exists $\rho_{0}(p)>0$ such that

$$
Q_{\varepsilon}(\rho):=\sup _{0<\varepsilon \leq 1} P\left(\varepsilon^{-2}\left\|D u^{\varepsilon}(t, x)\right\|_{\mathcal{H}_{T}}^{2} \leq \rho\right) \leq C_{p} \rho^{p}, \quad \forall \rho \in\left(0, \rho_{0}(p)\right) .
$$

For any $\rho, \sigma>0$ such that $\rho^{\sigma}<t$, we have that

$$
\begin{aligned}
\int_{0}^{t}\left\|\varepsilon^{-1} D_{s, \bullet} u^{\varepsilon}(t, x)\right\|_{\mathcal{H}}^{2} \mathrm{~d} s \geq & \int_{t-\rho^{\sigma}}^{t}\left\|\varepsilon^{-1} D_{s, \bullet} u^{\varepsilon}(t, x)\right\|_{\mathcal{H}}^{2} \mathrm{~d} s \\
\geq & \frac{1}{2} \int_{t-\rho^{\sigma}}^{t}\left\|K_{t-s}(x-\bullet) \theta\left(u^{\varepsilon}(t, x)\right)\right\|_{\mathcal{H}}^{2} \mathrm{~d} s \\
& -\int_{t-\rho^{\sigma}}^{t}\left\|\varepsilon^{-1} D_{s, \bullet} u^{\varepsilon}(t, x)-K_{t-s}(x-\bullet) \theta\left(u^{\varepsilon}(t, x)\right)\right\|_{\mathcal{H}}^{2} \mathrm{~d} s .
\end{aligned}
$$

So,

$$
Q_{\varepsilon}(\rho) \leq Q_{\varepsilon, 1}(\rho)+Q_{\varepsilon, 2}(\rho)
$$

with

$$
\begin{aligned}
& Q_{\varepsilon, 1}(\rho)=P\left(\int_{t-\rho^{\sigma}}^{t}\left\|\varepsilon^{-1} D_{s, \bullet} u^{\varepsilon}(t, x)-K_{t-s}(x-\bullet) \theta\left(u^{\varepsilon}(t, x)\right)\right\|_{\mathcal{H}}^{2} \mathrm{~d} s \geq \rho\right), \\
& Q_{\varepsilon, 2}(\rho)=P\left(\int_{t-\rho^{\sigma}}^{t}\left\|K_{t-s}(x-\bullet) \theta\left(u^{\varepsilon}(t, x)\right)\right\|_{\mathcal{H}}^{2} \mathrm{~d} s \leq 4 \rho\right) .
\end{aligned}
$$

Arguing as in (3.5) together with Gronwall's lemma allow to prove that, for any $p \geq 2$, there exists a positive constant $c_{p}$ such that

$$
\sup _{(\tau, x) \in\left[t-\rho^{\sigma}, t\right] \times \mathbb{R}^{d}} \mathbf{E}\left[\left|\int_{t-\rho^{\sigma}}^{t}\left\|D_{s, \bullet} u^{\varepsilon}(\tau, x)\right\|_{\mathcal{H}}^{2} \mathrm{~d} s\right|^{p}\right] \leq c_{p}\left[\int_{0}^{\rho^{\sigma}} \mathrm{d} s \int_{\mathbb{R}^{d}} \mu(\mathrm{d} \xi)\left|\mathcal{F} K_{s}(\bullet)(\xi)\right|^{2}\right]^{p} .
$$


Chebychev's and Burkholder's inequalities, Parseval's identity, Schwartz's and Hölder's inequalities, the condition on $\theta^{\prime}$ and this last estimate yield, for $\lambda \in[1, \infty)$,

$$
\begin{aligned}
Q_{\varepsilon, 1}(\rho) \leq & C \rho^{-\lambda} \mathbf{E}\left[\int_{t-\rho^{\sigma}}^{t}\left\|\int_{r}^{t} \int_{\mathbb{R}^{d}} K_{t-s}(x-y) D_{r, \bullet} u^{\varepsilon}(s, y) \theta^{\prime}\left(u^{\varepsilon}(s, y)\right) W(\mathrm{~d} s, \mathrm{~d} y)\right\|_{\mathcal{H}}^{2} \mathrm{~d} r\right]^{2 \lambda} \\
\leq & C \rho^{-\lambda}\left[\int_{0}^{\rho^{\sigma}} \mathrm{d} s \int_{\mathbb{R}^{d}} \mu(\mathrm{d} \xi)\left|\mathcal{F} K_{s}(\bullet)(\xi)\right|^{2}\right]^{2 \lambda} \\
= & C \rho^{-\lambda}\left[\int_{0}^{\rho^{\sigma}} \mathrm{d} s \int_{\{|\xi| \leq k\}} \mu(\mathrm{d} \xi) \exp \left\{-2 s|\xi|^{\alpha}\left(1+|\xi|^{2}\right)^{\frac{\beta}{2}}\right\}\right. \\
& \left.+\int_{0}^{\rho^{\sigma}} \mathrm{d} s \int_{\{|\xi|>k\}} \mu(\mathrm{d} \xi) \exp \left\{-2 s|\xi|^{\alpha}\left(1+|\xi|^{2}\right)^{\frac{\beta}{2}}\right\}\right]^{2 \lambda} .
\end{aligned}
$$

Then, since $\mathrm{e}^{x} \leq 1,1-\mathrm{e}^{-x} \leq 1$ and $1-\mathrm{e}^{-x} \leq x$, for all $x \geq 0$, using (2.1) and (2.4), we get, for $\hat{q}=1-q$,

$$
Q_{\varepsilon, 1}(\rho) \leq C \rho^{-\lambda}\left[\rho^{\sigma}+\rho^{\hat{q} \sigma} \int_{\{|\xi|>k\}} \frac{\mu(\mathrm{d} \xi)}{\left(1+|\xi|^{2}\right)^{(1-\hat{q}) \frac{\alpha+\beta}{2}}}\right]^{2 \lambda} \leq C \rho^{-\lambda+2 \lambda \sigma \hat{q}}=C \rho^{\lambda(2 \sigma \hat{q}-1)} .
$$

On the other hand, the properties of the Fourier transform, the fact that $\frac{1}{x}\left(1-\mathrm{e}^{-x}\right) \geq \frac{1}{1+x}$, for all $x>0$, and (2.1), imply

$$
\begin{aligned}
& \int_{t-\rho^{\sigma}}^{t}\left\|K_{t-s}(x-\bullet) \theta\left(u^{\varepsilon}(t, x)\right)\right\|_{\mathcal{H}}^{2} \mathrm{~d} s \geq \theta_{0}^{2} \int_{0}^{\rho^{\sigma}} \mathrm{d} s \int_{\mathbb{R}^{d}} \mu(\mathrm{d} \xi)\left|\mathcal{F} K_{s}(\bullet)(\xi)\right|^{2} \\
& \quad=\theta_{0}^{2} \int_{0}^{\rho^{\sigma}} \mathrm{d} s \int_{\mathbb{R}^{d}} \mu(\mathrm{d} \xi) \exp \left\{-2 s|\xi|^{\alpha}\left(1+|\xi|^{2}\right)^{\frac{\beta}{2}}\right\} \geq \theta_{0}^{2} \int_{\mathbb{R}^{d}} \frac{1-\mathrm{e}^{-2 \rho^{\sigma}|\xi|^{\alpha}\left(1+|\xi|^{2}\right)^{\frac{\beta}{2}}}}{2|\xi|^{\alpha}\left(1+|\xi|^{2}\right)^{\frac{\beta}{2}}} \mu(\mathrm{d} \xi) \\
& \quad \geq \theta_{0}^{2} \rho^{\sigma} \int_{\{|\xi| \leq k\}} \frac{1}{1+2 \rho^{\sigma}|\xi|^{\alpha}\left(1+|\xi|^{2}\right)^{\frac{\beta}{2}}} \mu(\mathrm{d} \xi) \geq \frac{\theta_{0}^{2} \rho^{\sigma}}{1+2 t k^{\alpha}\left(1+k^{2}\right)^{\frac{\beta}{2}}} \int_{\{|\xi| \leq k\}} \mu(\mathrm{d} \xi) \\
& \quad \geq C \theta_{0}^{2} \rho^{\sigma} .
\end{aligned}
$$

Choosing $\sigma \in(0,1)$, there exists $\rho_{0}>0$ such that, for any $\rho \in\left(0, \rho_{0}\right), Q_{\varepsilon, 2}(\rho)=0$. Moreover, recalling that $\hat{q}=1-q$ and $q \in\left(0, \frac{1}{2}\right)$, if $\sigma \in\left(\frac{1}{2 \hat{q}}, 1\right)$, the inequality (4.2) is satisfied.

Set

$$
\tilde{W}(t, x)=\operatorname{sgn}\left(x_{1} \times \cdots \times x_{n}\right) \int_{0}^{t} \int_{R(x)} W(\mathrm{~d} s, \mathrm{~d} x), \quad(t, x) \in[0, T] \times \mathbb{R}^{d} .
$$

Classical results on Gaussian processes (see, for instance, Thm. 3.4.12 in [15]) show that, for $\nu \in\left[0, \frac{1}{2}\right.$ ), the family $\{\varepsilon \tilde{W}, \varepsilon>0\}$ satisfies a large deviation principle on $\mathcal{C}^{\nu, \nu}\left([0, T] \times \mathbb{R}^{d} ; \mathbb{R}\right)$ with rate function

$$
\bar{I}(\bar{h})= \begin{cases}\frac{1}{2}\|\bar{h}\|_{H}^{2}, & \text { if } \bar{h} \in H \\ +\infty, & \text { otherwise. }\end{cases}
$$

Remark that, if $I_{T}(h)=\frac{1}{2}\|h\|_{\mathcal{H}_{T}}^{2}$ for $h \in \mathcal{H}_{T}$, then $\bar{I}(\bar{h})=I_{T}(h)$ for $\bar{h} \in H$.

Proposition 4.5. Assume (H1) and (2.4) for some $q \in\left(0, \frac{1}{2}\right)$. Then, the law of the solution $u^{\varepsilon}(t, x)$ obeys a large deviation principle with rate function

$$
I(y)=\inf \left\{\frac{1}{2}\|\bar{h}\|_{H}^{2}, \Psi^{\bar{h}}(t, x)=y\right\} .
$$


Proof. This result can be proved in a very similar way to [28]. The details of the proof of this proposition may be checked in a forthcoming paper. However, we give a sketch of the proof in the Appendix (see Sect. 4).

Lemma 4.6. Assume (H1) and (2.3). Then, the application $\bar{h} \in\{\bar{I} \leq a\} \longmapsto \Psi^{\bar{h}}$ is infinitely differentiable.

Proof. We give some initial ideas of the proof. The assumptions on the coefficients, Hölder's inequality and Gronwall's lemma imply, for $\bar{h}, \bar{k} \in H$,

$$
\sup _{(t, x) \in[0, T] \times \mathbb{R}^{d}}\left|\Psi^{\bar{h}}(t, x)\right| \leq C \quad \text { and } \quad \sup _{(t, x) \in[0, T] \times \mathbb{R}^{d}}\left|\Psi^{\bar{h}+\bar{k}}(t, x)-\Psi^{\bar{h}}(t, x)\right| \leq C\|\bar{k}\|_{H} .
$$

The Fréchet derivative is given by $D \Psi^{\bar{h}}(t, x)(\bar{k})=\left\langle\Xi_{\cdot, \bullet}^{\bar{h}}(t, x), k\right\rangle_{\mathcal{H}_{T}}$ with

$$
\begin{aligned}
\left\langle\Xi_{r, \bullet}^{\bar{h}}(t, x), \zeta\right\rangle_{\mathcal{H}} & =\Xi_{r, \zeta}^{\bar{h}}(t, x) \\
& =\left\langle K_{t-r}(x-\bullet) \theta\left(\Psi^{\bar{h}}(r, \bullet)\right), \zeta\right\rangle_{\mathcal{H}}+\left\langle K_{t-.}(x-\bullet) \theta^{\prime}\left(\Psi^{\bar{h}}(\cdot, \bullet)\right) \Xi_{r, \zeta}^{\bar{h}}(\cdot, \bullet), h\right\rangle_{\mathcal{H}_{T}},
\end{aligned}
$$

$\zeta \in \mathcal{H}$. So, Fubini's theorem implies

$$
D \Psi^{\bar{h}}(t, x)(\bar{k})=\left\langle K_{t-.}(x-\bullet) \theta\left(\Psi^{\bar{h}}(\cdot, \bullet)\right), k\right\rangle_{\mathcal{H}_{T}}+\left\langle K_{t-.}(x-\bullet) \theta^{\prime}\left(\Psi^{\bar{h}}(\cdot, \bullet)\right) D \Psi^{\bar{h}}(\cdot, \bullet)(\bar{k}), h\right\rangle_{\mathcal{H}_{T}} .
$$

Now, it is easy but tedious to check, using the assumptions on $\theta$, the properties of $K$, Hölder's inequality and Gronwall's lemma, that the application $\bar{h} \in\{\bar{I} \leq a\} \longmapsto \Psi^{\bar{h}}$ is Fréchet differentiable and $D \Psi^{\bar{h}}(t, x)$ its Fréchet derivative. The proof that it is infinitely Fréchet differentiable is a classical generalization.

Set

$$
\hat{u}_{\bar{h}}^{\varepsilon}(t, x):=u^{\varepsilon}(t, x)\left(\omega+\frac{\bar{h}}{\varepsilon}\right)=u^{1}(t, x)(\varepsilon \omega+\bar{h}), \quad \bar{h} \in H .
$$

This process satisfies the following stochastic partial differential equation

$$
\hat{u}_{\bar{h}}^{\varepsilon}(t, x)=\varepsilon \int_{0}^{t} \int_{\mathbb{R}^{d}} K_{t-s}(x-y) \theta\left(\hat{u}_{\bar{h}}^{\varepsilon}(s, y)\right) W(\mathrm{~d} s, \mathrm{~d} y)+\left\langle K_{t-.}(x-\bullet) \theta\left(\hat{u}_{\bar{h}}^{\varepsilon}(\cdot, \bullet)\right), h\right\rangle_{\mathcal{H}_{T}} .
$$

By uniqueness of solution we have that $\hat{u}_{\bar{h}}^{0}(t, x)=\Psi^{\bar{h}}(t, x)$ and $\hat{u}_{0}^{\varepsilon}(t, x)=u^{\varepsilon}(t, x)$. As in (3.4) of Theorem 2.1, we can prove that

$$
\sup _{\varepsilon \in(0,1]} \sup _{(t, x) \in[0, T] \times \mathbb{R}^{d}} \mathbf{E}\left[\left|\hat{u}_{\bar{h}}^{\varepsilon}(t, x)\right|^{p}\right]<\infty, \quad \forall p \in[1,+\infty) .
$$

Gronwall's lemma and this last fact allow to check that

$$
\lim _{\varepsilon \downarrow 0} \mathbf{E}\left[\left|\hat{u}_{\bar{h}}^{\varepsilon}(t, x)-\Psi^{\bar{h}}(t, x)\right|^{p}\right]=0, \quad \forall p \in[1,+\infty) .
$$

Now consider the following Gaussian process $G^{\bar{h}}(t, x)$ satisfying

$$
G^{\bar{h}}(t, x)=\int_{0}^{t} \int_{\mathbb{R}^{d}} K_{t-s}(x-y) \theta\left(\Psi^{\bar{h}}(s, y)\right) W(\mathrm{~d} s, \mathrm{~d} y)+\left\langle K_{t-.}(x-\bullet) \theta^{\prime}\left(\Psi^{\bar{h}}(\cdot, \bullet)\right), h\right\rangle_{\mathcal{H}_{T}} .
$$

If we define

$$
\hat{U}_{\bar{h}}^{\varepsilon}(t, x)=\frac{\hat{u}_{\bar{h}}^{\varepsilon}(t, x)-\Psi^{\bar{h}}(t, x)}{\varepsilon}, \quad 0<\varepsilon \leq 1,
$$

we can observe that

$$
\lim _{\varepsilon \downarrow 0} \mathbf{E}\left[\left|\hat{U}_{\bar{h}}^{\varepsilon}(t, x)-G^{\bar{h}}(t, x)\right|^{p}\right]=0, \quad \forall p \in[1,+\infty),
$$


uniformly in $[0, T] \times \mathbb{R}^{d}$. Since $\Psi^{\bar{h}}$ is deterministic and $G^{\bar{h}}$ is Gaussian, it can be quickly generalized as follows

$$
\mathbb{D}^{\infty}-\lim _{\varepsilon \downarrow 0}\left[\hat{U}_{\bar{h}}^{\varepsilon}(t, x)-G^{\bar{h}}(t, x)\right]=0 .
$$

Indeed, it can be proved using the hypothesis on $\theta$ and the spectral measure $\mu$ and by means of an argument based on Hölder's and Burkholder's inequalities and Gronwall's lemma.

Now we have all the requirements to prove Theorem 2.3.

Proof of Theorem 2.3. In order to check the upper bound we apply Lemma 4.3, Lemma 4.4 and Proposition 4.5 to $X^{\varepsilon}=u^{\varepsilon}(t, x)$ at a fixed point $(t, x) \in[0, T] \times \mathbb{R}^{d}$. Indeed, these three results ensure that the assumptions (i), (ii) and (iii) of Proposition 4.1 are satisfied and consequently

$$
\lim _{\varepsilon \downarrow 0} \varepsilon^{2} \log p_{t, x}^{\varepsilon}(t, x) \leq-d_{t}^{2}(x, y),
$$

with

$$
d_{t}^{2}(x, y)=\inf \left\{\frac{1}{2}\|\bar{h}\|_{H}^{2}, \Psi^{\bar{h}}(t, x)=y\right\} .
$$

Now we prove the lower bound. The assumptions of Proposition 4.2 are satisfied for $X^{\varepsilon}=u^{\varepsilon}(t, x), \Lambda(\bar{h})=$ $\Psi^{\bar{h}}(t, x)$ and $Z(\bar{h})=G^{\bar{h}}(t, x)$. So, we have that

$$
\lim _{\varepsilon \downarrow 0} \varepsilon^{2} \log p_{t, x}^{\varepsilon}(t, x) \geq-\tilde{d}_{t}^{2}(x, y),
$$

with

$$
\tilde{d}_{t}^{2}(x, y)=\inf \left\{\frac{1}{2}\|\bar{h}\|_{H}^{2}, \Psi^{\bar{h}}(t, x)=y, \gamma_{t, x}^{G}(\bar{h})>0\right\},
$$

where $\gamma_{t, x}^{G}(\bar{h})=\left\langle D G^{\bar{h}}(t, x), D G^{\bar{h}}(t, x)\right\rangle_{\mathcal{H}_{T}}$. Then, if we check that, for any $\bar{h} \in H, \gamma_{t, x}^{G}(\bar{h})>0$, for any fixed $(t, x) \in[0, T] \times \mathbb{R}^{d}$, since $d_{t}^{2}(x, y)=\tilde{d}_{t}^{2}(x, y)$, we will have concluded the proof of this theorem.

By uniqueness of solution we have that $D G^{\bar{h}}(t, x)=D \Psi^{\bar{h}}(t, x)$. So,

$$
\begin{aligned}
\gamma_{t, x}^{G}(\bar{h}) & =\gamma_{t, x}^{\Psi}(\bar{h})=\left\langle D \Psi^{\bar{h}}(t, x), D \Psi^{\bar{h}}(t, x)\right\rangle_{\mathcal{H}_{T}}=\int_{0}^{t}\left\|\Xi_{r, \bullet}^{\bar{h}}(t, x)\right\|_{\mathcal{H}}^{2} \mathrm{~d} r \\
& \geq \int_{t-\rho}^{t}\left\|\Xi_{r, \bullet}^{\bar{h}}(t, x)\right\|_{\mathcal{H}}^{2} \mathrm{~d} r \geq \frac{1}{2} \int_{t-\rho}^{t}\left\|K_{t-r}(x-\bullet) \theta\left(\Psi^{\bar{h}}(r, \bullet)\right)\right\|_{\mathcal{H}}^{2} \mathrm{~d} r-A_{t, x}(\rho),
\end{aligned}
$$

with

$$
\begin{aligned}
A_{t, x}(\rho) & =\int_{t-\rho}^{t}\left\|\Xi_{r, \bullet}^{\bar{h}}(t, x)-K_{t-r}(x-\bullet) \theta\left(\Psi^{\bar{h}}(r, \bullet)\right)\right\|_{\mathcal{H}}^{2} \mathrm{~d} r \\
& =\int_{t-\rho}^{t}\left\|\left\langle K_{t-\cdot}(x-\bullet) \theta^{\prime}\left(\Psi^{\bar{h}}(\cdot, \bullet)\right) \Xi_{r, *}^{\bar{h}}(\cdot, \bullet), h\right\rangle_{\mathcal{H}_{T}}\right\|_{\mathcal{H}}^{2} \mathrm{~d} r .
\end{aligned}
$$

The same argument as in (4.3) allows to prove that

$$
\frac{1}{2} \int_{t-\rho}^{t}\left\|K_{t-r}(x-\bullet) \theta\left(\Psi^{\bar{h}}(r, \bullet)\right)\right\|_{\mathcal{H}}^{2} \mathrm{~d} r \geq C \theta_{0}^{2} \rho .
$$

We now deal with $A_{t, x}(\rho)$. Let $\left\{e_{j}, j \geq 0\right\}$ be a CONS of $\mathcal{H}$, then

$$
A_{t, x}(\rho)=A_{t, x}^{1}(\rho)+A_{t, x}^{2}(\rho),
$$


with

$$
\begin{aligned}
A_{t, x}^{1}(\rho)= & \int_{t-\rho}^{t} \sum_{j \geq 0} \mid \int_{r}^{t}\left\langle K_{t-s}(x-\bullet) \theta^{\prime}\left(\Psi^{\bar{h}}(s, \bullet)\right)\right. \\
& \left.\times\left\langle K_{s-r}(\bullet-*) \theta\left(\Psi^{\bar{h}}(r, *)\right), e_{j}\right\rangle_{\mathcal{H}}, h\right\rangle\left._{\mathcal{H}} \mathrm{d} s\right|^{2} \mathrm{~d} r, \\
A_{t, x}^{2}(\rho)= & \int_{t-\rho}^{t} \sum_{j \geq 0} \mid \int_{r}^{t}\left\langleK _ { t - s } ( x - \bullet ) \theta ^ { \prime } ( \Psi ^ { \overline { h } } ( s , \bullet ) ) \left[\Xi_{r, e_{j}}^{\bar{h}}(s, \bullet)\right.\right. \\
& \left.\left.-\left\langle K_{s-r}(\bullet-*) \theta\left(\Psi^{\bar{h}}(r, *)\right), e_{j}\right\rangle_{\mathcal{H}}\right], h\right\rangle\left._{\mathcal{H}} \mathrm{d} s\right|^{2} \mathrm{~d} r .
\end{aligned}
$$

Using Cauchy's inequality, (H1), Parseval's identity, (2.1), Fubini's theorem and the properties of $K$, and arguing as by $Q_{\varepsilon, 1}(\rho)$ with $\lambda=1$ in Lemma 4.4 , we have that

$$
\begin{aligned}
A_{t, x}^{1}(\rho) & \leq C\|h\|_{\mathcal{H}_{T}}^{2} \int_{t-\rho}^{t} \mathrm{~d} r \int_{r}^{t} \mathrm{~d} s \int_{\mathbb{R}^{d}} \mu(\mathrm{d} \xi)\left|\mathcal{F} K_{t-s}(\bullet)(\xi)\right|^{2} \sup _{x \in \mathbb{R}^{d}}\left\|K_{s-r}(x-*)\right\|_{\mathcal{H}}^{2} \\
& \leq C\|h\|_{\mathcal{H}_{T}}^{2} \rho^{2 \hat{q}}
\end{aligned}
$$

with $\hat{q}=1-q$.

In a similar way we can obtain that

$$
A_{t, x}^{2}(\rho) \leq C\|h\|_{\mathcal{H}_{T}}^{2} \int_{t-\rho}^{t} \mathrm{~d} s\left[\int_{\mathbb{R}^{d}} \mu(\mathrm{d} \xi)\left|\mathcal{F} K_{t-s}(\bullet)(\xi)\right|^{2}\right] \sup _{r \leq s} \sup _{x \in \mathbb{R}^{d}} A_{s, x}(\rho) .
$$

Then, Gronwall's lemma implies

$$
A_{t, x}(\rho) \leq C \rho^{2 \hat{q}} .
$$

So, there exists $\rho_{0}>0$ such that for any $\rho \in\left(0, \rho_{0}\right)$,

$$
\gamma_{t, x}^{\Psi}(\bar{h}) \geq C \theta_{0}^{2} \rho-C \rho^{2 \hat{q}}>0 .
$$

Remark 4.7. In the sequel we will give some ideas in order to justify that $d_{t}^{2}(x, y)$ is finite. We could suppose that the topological support of the probability distribution of $u^{\varepsilon}(t, x)$ satisfies

$$
\overbrace{\text { support }\left[P \circ\left(u^{\varepsilon}(t, x)\right)^{-1}\right]}^{\circ}=\left\{y \in \mathbb{R} \text {; such that } \exists \bar{h}_{1} \in H \text { with } \Psi^{\bar{h}_{1}}(t, x)=y\right\} .
$$

By [17], the set support $\left[P \circ\left(u^{\varepsilon}(t, x)\right)^{-1}\right]$ is a closed interval of $\mathbb{R}$. Then, assuming the same hypothesis as in Theorem 2.3 and that $\theta$ is positive (the case $\theta$ negative is analogous), we have that

$$
\left\{y \in \mathbb{R}, d_{t}^{2}(x, y)<\infty\right\}=\mathbb{R} .
$$

Indeed, it is easy to see that

$$
\left\langle K_{t-\cdot}(x-\bullet) \theta\left(\Psi^{\bar{h}}(\cdot, \bullet)\right), K_{t-.}(x-\bullet)\right\rangle_{\mathcal{H}_{T}} \geq C \rho \theta_{0} .
$$

Taking $\tau>0$, for any $y \in \mathbb{R}$, we can define

$$
\bar{h}_{1,1}(s, z)=\frac{(|y|+\tau) K_{t-s}(x-z)}{C \rho \theta_{0}}=-\bar{h}_{1,2}(s, z) .
$$


Then,

$$
\Psi^{\bar{h}_{1,2}}(t, x)<y<\Psi^{\bar{h}_{1,1}}(t, x) .
$$

As the set support $\left[P \circ\left(u^{\varepsilon}(t, x)\right)^{-1}\right]$ is a closed interval of $\mathbb{R}$, we have that for any $y \in \mathbb{R}$, there exists $\bar{h}_{1} \in H$ such that $\Psi^{\bar{h}_{1}}(t, x)=y$ and, so, $d_{t}^{2}(x, y)$ is finite.

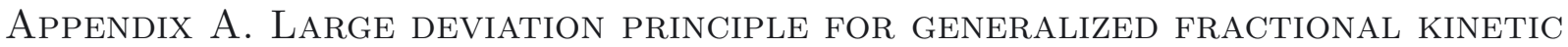 EQUATIONS}

In this appendix we give some details of the proof of Proposition 4.5. It is similar to the proof given in [28]. The basic ideas are based on a classical result of Azencott [6] that allow us to beyond a large deviation principle from our initial perturbed Gaussian process to $u^{\varepsilon}$.

Theorem A.1. Let $\left(E_{i}, d_{i}\right), i=1,2$, be two Polish spaces and $X_{i}: \Omega \rightarrow E_{i}, \varepsilon>0, i=1,2$, be two families of random variables. Suppose the following requirements:

- Step 1. $\left\{X_{1}^{\varepsilon}, \varepsilon>0\right\}$ obeys a large deviation principle with the rate function $I_{1}: E_{1} \rightarrow[0, \infty]$.

- Step 2. There exists a function $S:\left\{I_{1}<\infty\right\} \rightarrow E_{2}$ such that, for every $a<\infty$, the function

$$
S:\left\{I_{1} \leq a\right\} \rightarrow E_{2}
$$

is continuous.

- Step 3. For every $R, \rho, a>0$, there exist $\theta>0$ and $\varepsilon_{0}>0$ such that, for $h \in E_{1}$ satisfying $I_{1}(h) \leq a$ and $\varepsilon \leq \varepsilon_{0}$, we have

$$
P\left\{d_{2}\left(X_{2}^{\varepsilon}, S(h)\right) \geq \rho, d_{1}\left(X_{1}^{\varepsilon}, h\right)<\theta\right\} \leq \exp \left(-\frac{R}{\varepsilon^{2}}\right) .
$$

Then, the family $\left\{X_{2}^{\varepsilon}, \varepsilon>0\right\}$ obeys a large deviation principle with the rate function

$$
I_{2}(\phi)=\inf \left\{I_{1}(h): S(h)=\phi\right\} .
$$

We know that the family $\{\varepsilon \tilde{W}, \varepsilon>0\}$ satisfies a large deviation principle on $\mathcal{C}^{\nu, \nu}\left([0, T] \times \mathbb{R}^{d} ; \mathbb{R}\right)$ (Step 1$)$. So, in order to prove that $u^{\varepsilon}$ satisfies a large deviation principle we only need to check the existence of a function $S$ and (A.1) (Step 2 and 3).

First of all we introduce some basic notations. We write, for $\phi, \phi^{\prime}:[0 . T] \times \mathbb{R}^{d} \rightarrow \mathbb{R}$,

$$
\begin{aligned}
\|\phi\|_{\infty} & =\sup \left\{|\phi(t, x)|, \quad(t, x) \in[0 . T] \times \mathbb{R}^{d}\right\}, \\
\|\phi\|_{\gamma_{1}, \gamma_{2}} & =\sup \left\{\frac{|\phi(t, x)-\phi(s, y)|}{|t-s|^{\gamma_{1}}+\|x-y\|^{\gamma_{2}}},(t, x) \neq(s, y) \in[0 . T] \times \mathbb{R}^{d}\right\}, \\
d_{\gamma_{1}, \gamma_{2}}\left(\phi, \phi^{\prime}\right) & =\left\|\phi-\phi^{\prime}\right\|_{\infty}+\left\|\phi-\phi^{\prime}\right\|_{\gamma_{1}, \gamma_{2}} .
\end{aligned}
$$

Then, we define the topology of $\left(\gamma_{1}, \gamma_{2}\right)$-Hölder convergence on $[0 . T] \times \mathbb{R}^{d}$ by means of $d_{\gamma_{1}, \gamma_{2}}$. Let $\mathcal{C}^{\gamma_{1}, \gamma_{2}}\left(D_{T}^{d}\right)$ be the set of functions $\phi:[0 . T] \times \mathbb{R}^{d} \rightarrow \mathbb{R}$ such that $\|\phi\|_{\infty}+\|\phi\|_{\gamma_{1}, \gamma_{2}}<\infty$.

Step 2. Recall that for any $\bar{h} \in H$, we consider the deterministic evolution equation

$$
\Psi^{\bar{h}}(t, x)=\left\langle K_{t-.}(x-\bullet) \theta\left(\Psi^{\bar{h}}(\cdot, \bullet)\right), h\right\rangle_{\mathcal{H}_{T}}, \quad \forall(t, x) \in \mathbb{R}_{+} \times \mathbb{R}^{d} .
$$

Similar arguments to Proposition 2.2 in [25] prove that, for any $a \in[0,+\infty)$, we have

$$
\sup _{\bar{h}: \bar{I}(\bar{h}) \leq a} \sup _{(t, x) \in[0, T] \times \mathbb{R}^{d}}\left|\Psi^{\bar{h}}(t, x)\right| \leq C .
$$


Following the ideas given in Theorem 3.2 and Proposition 3.3 in [25] we can obtain that, for any $\rho_{1}<\frac{1-q}{2}$ and $\rho_{2}<1-q$, there exists a constant $C$ such that, for any $(t, x),(s, z) \in[0, T] \times \mathbb{R}^{d}$,

$$
\sup _{\bar{h}: \bar{I}(\bar{h}) \leq a}\left|\Psi^{\bar{h}}(t, x)-\Psi^{\bar{h}}(s, z)\right| \leq \begin{cases}C\left(|t-s|^{\gamma_{1}}+|x-z|^{\gamma_{2}}\right), & \text { if } \alpha+\beta \geq 2, \\ C\left(|t-s|^{\gamma_{1}}+|x-z|^{\frac{1}{2} \gamma_{2}(\alpha+\beta)}\right), & \text { if } \alpha+\beta<2 .\end{cases}
$$

Finally, as in Theorem 4.2 in [28], we can get that, for every $a>0$, the map $\bar{h} \rightarrow \Psi^{\bar{h}}$ is continuous from $\{\bar{I} \leq a\}$ to $\mathcal{C}^{\rho_{1}, \rho_{2}}\left([0, T] \times \mathbb{R}^{d}\right)$, for the corresponding values of $\left(\rho_{1}, \rho_{2}\right)$, and where $\{\bar{I} \leq a\}$ is endowed with the topology of uniform convergence. We argue as in Theorem $4.2[28]$ but considering first $x \in[-N, N]^{d}$ and then tending $N$ to infinity.

Step 3. Here we have followed the approach of Freidlin and Wentzell [18] for diffusion process (see also Dembo and Zeitouni [14]). In fact, the proof is easier than in Proposition 5.1 [28] since the fundamental solution in [28] is more general.

\section{REFERENCES}

[1] J.M. Angulo, V.V. Anh, R. McVinish and M.D. Ruiz-Medina, Fractional kinetic equations driven by Gaussian or infinitely divisible noise. Adv. Appl. Prob. 37 (2005) 366-392.

[2] J.M. Angulo, M.D. Ruiz-Medina, V.V. Anh and W. Grecksch, Fractional diffusion and fractional heat equation. Adv. Appl. Prob. 32 (2000) 1077-1099.

[3] V.V. Ahn, J.M. Angulo and M.D. Ruiz-Medina,. Possible long-range dependence in fractional randoms fields. J. Statist. Plan. Infer. 80 (1999) 95-110

[4] V.V. Anh and N.N. Leonenko, Spectral analysis of fractional kinetic equation with random data. J. Stat. Phys. 104 (2001) 1349-1387.

[5] V.V. Anh and N.N. Leonenko, Renormalization and homogenization of fractional diffusion equations with random data. Probab. Theory Related Fields 124 (2002) 381-408.

[6] R. Azencott, Grandes déviations et applications. École d'Été de Probabilités de Saint-Flour VIII-1978. In vol. 774 of Lect. Notes Math. Springer-Verlag, Berlin (1980) 1-176.

[7] L. Boulanba, M. Eddahbi and M. Mellouk, Fractional SPDEs driven by spatially correlated noise: existence of the solution and smoothness of its density. Osaka J. Math. 47 (2010) 41-65.

[8] X. Cabré and M. Sanchón, Semi-stable and extremal solutions of reaction equations involving the p-Laplacian. Commun. Pure Appl. Anal. 6 (2007) 43-67.

[9] L. Caffarelli and L. Silvestre, An extension problem related to the fractional Laplacian. Comm. Partial Differ. Eq. 32 (2007) 1245-1260.

[10] R.C. Dalang and N.E. Frangos, The stochastic wave equation in two spatial dimensions. Ann. Probab. 26 (1998) 187-212.

[11] R.C. Dalang, Extending the martingale measure stochastic integral with applications to spatially homogeneous spde's. Electron. J. Probab. 4 (1999) 29.

[12] R. Dautray and J.L. Lions, Mathematical Analysis and Numerical Methods for Science and Technology. 2. Springer, Berlin (1995).

[13] L. Debbi and M. Dozzi, On the solutions of nonlinear stochastic fractional partial differential equations in one spatial dimension. Stochastic Processes Appl. 115 (2005) 1764-1781.

[14] A. Dembo and O. Zeitouni, Large deviations techniques and applications. Jones and Barlett Publishers, Boston (1983).

[15] J.D. Deuschel and D.W. Stroock, Large deviations. In vol. 137 of Pure Appl. Math. Academic Press, Boston (1989).

[16] E. Dibenedetto, U. Gianazza and V. Vespri, Intrinsic Harnack estimates for nonnegative local solutions of degenerate parabolic equations. Electron. Res. Announc. Amer. Math. Soc. 12 (2006) 95-99.

[17] S. Fang, Une inégalité isopérimétrique sur l'espace de Wiener. Bull. Sc. Math. 2e série. 112 (1988) 345-355.

[18] M.I. Freidlin and A.D. Wentzell, Random perturbation of dynamical systems. Springer-Verlag, New York (1984).

[19] K.J. Hochberg, A signed measure on path space related to Wiener measure. Ann. Probab. 6 (1978) 433-458.

[20] B. Jourdain, S. Méléard and W.A. Woyczynski, Probabilistic approximation and inviscid limits for one-dimensional fractional conservation laws. Bernoulli 11 (2005) 689-714.

[21] B. Jourdain, S. Méléard and W.A. Woyczynski, A probabilistic approach for nonlinear equations involving the fractional Laplacian and a singular operator. Potential Anal. 23 (2005) 55-81.

[22] V.Y. Krylov, Some properties of the distribution corresponding to the equation $\frac{\partial u}{\partial t}=(-1)^{q+1} \frac{\partial^{2} q}{\partial x^{2} q}$. Soviet Math. Dokl. 1 (1960) 760-763.

[23] B.B. Mandelbrot, and J.W. Van Ness, Fractional Brownian motion, fractional noises and applications. SIAM Rev. 10 (1968) 422-437.

[24] D. Márquez-Carreras, Contribució a l'estudi de les equacions en derivades parcials estocàstiques. Ph.D. thesis (1998). 
[25] D. Márquez-Carreras, Generalized fractional kinetic equations: another point of view. Adv. Appl. Prob. 41 (2009)893-910.

[26] D. Márquez-Carreras, M. Mellouk and M. Sarrà, On stochastic partial differential equations with spatially correlated noise: smoothness of the law. Stochastic Processes Appl. 93 (2001) 269-284.

[27] D. Márquez-Carreras and M. Sanz-Solé, Small perturbations in a hyperbolic stochastic partial differential equation. Stochastic Processes Appl. 68 (1997) 133-154.

[28] D. Márquez-Carreras and M. Sarrà, Large deviation principle for a stochastic heat equation with spatially correlated noise. Electronic J. Prob. 8 (2003) 1-39.

[29] S. Micu, and E. Zuazua, On the controllability of a fractional order parabolic equation. SIAM J. Control Optim. 44 (2006) 1950-1972

[30] A. Millet and M. Sanz-Solé, A stochastic wave equation in two space dimension: Smoothness of the law. Ann. Probab. 27-2 (1999) 803-844.

[31] D. Nualart, Analysis on Wiener Space and Anticipating stochastic Calculus. École d'Été de Probabilités de Saint-Flour XXV1995, in vol. 1690 of Lect. Notes Math. Springer-Verlag (1998) 123-227.

[32] D. Nualart, Malliavin Calculus and Related Topics. Springer-Verlag (1995).

[33] D. Nualart and LL. Quer-Sardanyons, Existence and smoothness of the density for spatially homogeneous SPDEs. Potential Anal. 27 (2007) 281-299

[34] M.D. Ruiz-Medina and J.M. Angulo and V.V. Anh, Scaling limit solution of a fractional Burgers equation. Stochastic Processes Appl. 93 (2001) 285-300.

[35] S.G. Samko, A.A. Kilbas and O.I. Marichev, Fractional integrals and derivatives. Gordon and Breach Science Publishers, New York (1987).

[36] L. Schwartz, Théorie des Distributions. Hermann, Paris (1966).

[37] E.M. Stein, Singular integrals and differentiating properties of Functions. Princeton University Press, Princeton (1970).

[38] J.B. Walsh, An introduction stochastic partial differential equations. École d'été de probabilités de Saint Flour XIV 1984. In vol. 1180 of Lect. Notes Math. Springer, Berlin (1986) 265-439. 